\title{
Perceived acceptable uncertainty regarding comparability of endovascular treatment alone versus intravenous thrombolysis plus endovascular treatment
}

\author{
Johannes Kaesmacher (D) , ${ }^{1}$ Adnan Mujanovic (D) , 1,2 Kilian Treurniet, ${ }^{3}$ \\ Manon Kappelhof, ${ }^{3}$ Thomas R Meinel (10 ,' 2 Pengfei Yang, ${ }^{4}$ Jianmin Liu, ${ }^{4}$ \\ Yongwei Zhang, ${ }^{4}$ Wenjie Zi, ${ }^{5}$ Qingwu Yang, ${ }^{5}$ Raul G Nogueira, ${ }^{6}$ Kazumi Kimura, ${ }^{7}$ \\ Yuji Matsumaru, ${ }^{8}$ Kentaro Suzuki, ${ }^{7}$ Bernard Yan,, 10 Peter J Mitchell, ${ }^{10}$ \\ Zhongrong Miao, ${ }^{11}$ Yvo B W E M Roos, ${ }^{12}$ Charles B L M Majoie, ${ }^{3}$ Jan Gralla, ${ }^{1}$ \\ Jeffrey L Saver, ${ }^{13,14}$ Urs Fischer $^{2}$
}

- Additional supplemental material is published online only. To view, please visit the journal online (http://dx.doi. org/10.1136/neurintsurg2022-018665).

For numbered affiliations see end of article.

\section{Correspondence to} Mr Johannes Kaesmacher, University of Bern, Bern, Switzerland; johannes. kaesmacher@insel.ch

JK and AM are joint first authors.

$J G$, JLS and UF are joint senior authors.

Received 10 January 2022 Accepted 27 January 2022

Check for updates

(c) Author(s) (or their employer(s)) 2022. Re-use permitted under CC BY-NC. No commercial re-use. See rights and permissions. Published by BMJ.

To cite: Kaesmacher $J_{\text {, }}$ Mujanovic A, Treurniet $K_{\text {, }}$ et al. J Neurolntervent Surg Epub ahead of print: [please include Day Month Year]. doi:10.1136/

neurintsurg-2022-018665

\section{ABSTRACT}

Background Most trials comparing endovascular treatment (EVT) alone versus intravenous thrombolysis with alteplase (IVT) + EVT in directly admitted patients with a stroke are non-inferiority trials. However, the margin based on the level of uncertainty regarding noninferiority of the experimental treatment that clinicians are willing to accept to incorporate EVT alone into clinical practice remains unknown.

Objective To characterize what experienced stroke clinicians would consider an acceptable level of uncertainty for hypothetical decisions on whether to administer IVT or not before EVT in patients admitted directly to EVT-capable centers.

Methods A web-based, structured survey was distributed to a cross-section of 600 academic neurologists/neurointerventionalists. For this purpose, a response framework for a hypothetical trial comparing IVT+EVT (standard of care) with EVT alone (experimental arm) was designed. In this trial, a similar proportion of patients in each arm achieved functional independence at 90 days. Invited physicians were asked at what level of certainty they would feel comfortable skipping IVT in clinical practice, considering these hypothetical trial results.

Results There were 180 respondents (response rate: $30 \%$ ) and 165 with complete answers. The median chosen acceptable uncertainty suggesting reasonable comparability between both treatments was an absolute difference in the rate of day 90 functional independence of 3\% (mode 5\%, IQR 1-5\%), with higher chosen margins observed in interventionalists (aOR 2.20,95\% Cl 1.06 to 4.67).

Conclusion Physicians would generally feel comfortable skipping IVT before EVT at different certainty thresholds. Most physicians would treat with EVT alone if randomized trial data suggested that the number of patients achieving functional independence at 90 days was similar between the two groups, and one could be sufficiently sure that no more than 3 out of 100 patients would not achieve functional independence at 90 days due to skipping IVT.

\section{INTRODUCTION}

The aim of non-inferiority trials is to show that there is no relevant loss of efficacy of the experimental arm as compared with the control. ${ }^{1}$ For this purpose, a non-inferiority margin is used to assess whether the experimental treatment is better than, or at least preserves the effect of, the active control. $^{2-4}$

The non-inferiority margin is selected using one of four frameworks to define what fraction of the active comparator treatment effect the experimental treatment must be demonstrated to preserve: (1) indistinguishability, (2) reasonable comparability, (3) clinical acceptability considering other advantages, and (4) indirect demonstration of superiority over placebo. ${ }^{2-7}$ In the most stringent, indistinguishability approach, the non-inferiority margin is defined as the minimal clinical important difference (MCID) for the outcome. The MCID is the smallest change, meaningful to patients. ${ }^{8}$ However, sample sizes required to demonstrate indistinguishability are often infeasibly large. Therefore, trials may instead undertake the reasonable comparability approach, in which the non-inferiority margin is selected based on the level of uncertainty regarding non-inferiority of the experimental treatment that clinicians are willing to accept to proceed with incorporating the new treatment into clinical practice.

One treatment approach for ischemic stroke that is currently undergoing intensive investigation in randomized non-inferiority clinical trials is treating large vessel occlusions with endovascular treatment (EVT) alone (novel approach, EVT alone) compared with intravenous thrombolysis with alteplase (IVT) plus endovascular treatment (standard active treatment, IVT+EVT) in patients presenting directly at an EVT-capable center. None of the first six trials provided detailed clinical justification for the noninferiority margins they selected ${ }^{9-15}$ a common infelicity in design or reporting of non-inferiority trials. ${ }^{16}$ To interpret the results of these studies, it 
will be helpful to consider trial findings in the context of both the indistinguishability margin and the reasonable comparability margin for their functional independence endpoint (modified Rankin Scale score $0-2$ at 3 months). A prior study has indicated that the indistinguishability margin value is $1.3 \%,{ }^{17}$ but to our knowledge no study has yet been undertaken to define the reasonable comparability margin.

Only clinical judgment, not statistical analysis of past studies, can define what fraction of the active comparator treatment effect must be preserved for the experimental treatment to be considered reasonably comparable to the reference treatment. Establishing this value is critical to interpretation of the results of the EVT alone versus IVT+EVT trials. We therefore undertook an international survey study to characterize what experienced stroke clinicians would consider an acceptable level of uncertainty regarding hypothetical decisions on whether to skip or administer IVT before EVT in patients admitted directly to EVTcapable centers.

\section{METHODS}

Design

We designed a survey response framework for a hypothetical trial comparing an IV lytic drug followed by an endovascular intervention (standard of care) with endovascular intervention alone (experimental arm), in which a similar proportion of patients admitted directly to an EVT-capable center achieved functional independence at 90 days. Physicians were asked at what level of certainty they would feel comfortable skipping the IV lytic drug in clinical practice, considering these hypothetical trial results. The survey included 12 questions on three separate web pages. The first seven questions, together with the description and aim of the survey, were displayed on the first page and assessed baseline responder variables, including sex, training, geographic location, country, appointment level, annual mechanical thrombectomy volume of the center and time devoted to the care of patients with a stroke.

The eighth question was displayed on the second page and dealt with the non-inferiority margin or the perceived acceptable level of uncertainty using the following scenario:

'In an acute stroke trial, patients with a large vessel occlusion are randomized to an IV lytic drug followed by an endovascular intervention (standard of care) or the endovascular intervention alone (experimental arm). The endovascular intervention without the IV lytic drug yielded the same number of patients achieving functional independence (modified Rankin Score 0-2) at 90 days after stroke, but the degree of certainty of this result is limited by trial sample size. This uncertainty implies that, while it is most likely that skipping the drug has no effect on the outcome of patients, it is also possible that it improves or worsens the outcome to some degree. There are several pathophysiological arguments in favor and against using the IV lytic drug before the endovascular intervention (current standard of care). How much certainty would you deem sufficient to skip the IV lytic drug and treat patients with the endovascular intervention alone in clinical practice? I would feel comfortable skipping the IV lytic drug if the best estimate is that endovascular intervention alone yields the same number of independent outcomes, there is also a possibility that skipping IV lysis increases the number of independent outcomes, and I can be highly confident that, in the worst possible scenario, no more than X out of 100 patients treated with endovascular intervention alone will fail to regain functional independence due to skipping the IV lytic drug.'

Response options to replace the $\mathrm{X}$ in the text above, were integer values without decimals ranging from 0 to 100 percentage points. For visual guidance six potential answer values were displayed graphically on the same page, specifically including 0 as a legitimate response option (online supplemental figure 1).

Three additional questions were displayed on the last survey page that elicited information about respondents' history of participation in three types of salient randomized-controlled trials. Specifically responders were asked to state if they participated as an investigator in trials which compared: (1) IVT versus placebo; (2) EVT+best medical treatment versus best medical treatment only; and (3) EVT alone versus IVT+EVT (online supplemental table 1). The final 12th question addressed management of administering IVT in patients undergoing EVT. The full survey questionnaire can be found in the online supplemental appendix. Data are available from the corresponding author on a reasonable request, together with a research plan proposal.

\section{Distribution}

Before distribution of the survey, there was an internal pilot phase with 25 participants giving feedback on the functionality and comprehensiveness of the survey. After incorporating the feedback, the survey was distributed among non-interventional and interventional neurologists, non-interventional and interventional neuroradiologists affiliated with a university hospital or large tertiary stroke care center around the globe. Email addresses and baseline variables were extracted from publicly available information, including institutional home pages and published curricula vitae. Physicians from all continents were invited, while being cognizant of non-interventional and interventional counterpoises. Sampling focused on major cities with a high volume of patients with a stroke while still maintaining sufficient continental representation. The initial invitation was sent out to 600 participants. We replaced 25 non-functioning email addresses of potential participants, and in 19 cases, participants were replaced, because no alternative functioning email addresses could be found. The final target population constituted 600 invited participants with functioning email addresses. After the initial survey invitation on July 26, 2021, three reminders were sent out to non-responders, with each reminder being sent after a 7-day period. The survey was closed and the database locked on August 23, 2021. Ethical approval and patient consent were not sought for this study owing to its nature of a proposed hypothetical trial.

\section{Statistical analysis}

Data descriptives are displayed as $\mathrm{n} / \mathrm{N}$ (\%) or median (IQR). Bar charts are used to display frequency of selected acceptable uncertainties, with inputs $>10 \%$, summarized as $>10 \%$ for graphical purposes. Group comparisons were performed using standard univariable statistical measures (Mann-Whitney U test for ordinal variables, exact Fisher's test for categorical variables). Association of respondents' characteristics with selected acceptable uncertainty was additionally evaluated using logistic regression analyses. For logistic regression analyses, the acceptable uncertainty was defined as an independent variable and was split at different quartiles (primary analysis: median split, sensitivity analyses: $25 \%$ and $75 \%$ quartiles). Potential for response bias was evaluated comparing non-respondents and respondents, as well as comparing early and late responders (using a median split of respondent timing). Incomplete responses were rated as non-responders. Medical training was presented as a dichotomized variable encompassing interventional (interventional stroke neurologists and neuroradiologists) and non-interventional training (vascular stroke neurologists and 
diagnostic neuroradiologists). Goodness of fit for baseline variables was evaluated by comparing responses provided by the responders with those extracted from publicly available sources. $\kappa$ Values were calculated to evaluate agreement. $\kappa$ Values of $0.6-0.79,0.80-0.90$, and $>0.90$ were rated as moderate, strong, and almost perfect agreement, respectively, corresponding to $35-63 \%, 64-81 \%$, and $82-100 \%$ reliability of the data. All tests are two-sided and a p value $\leq 0.05$ was considered significant.

\section{RESULTS}

There were 180 survey respondents (response rate: $30 \%$ ), and 165 respondents completely answered all survey questions. Baseline characteristics of the respondents with complete answers are shown in table 1 . Almost three-quarters were male, and there was an equal representation of interventional and non-interventional subspecialties. Most respondents were at a senior appointment level and had $10-50 \%$ of their care time dedicated to patients with a stroke. When asked about IVT management in patients undergoing mechanical thrombectomy, 61.8\% stated they did not stop the full IVT dose unless there was a clear medical reason to do so, whereas others answered that they stopped IVT infusion if certain criteria were met.

\section{Representativeness}

Goodness of fit for baseline variables were 0.96 (95\% CI 0.91 to 1.00$), 0.69$ (95\% CI 0.58 to 0.79 ), 0.96 (95\% 0.92-1.00), 0.86 (95\% CI 0.79 to 0.96$)$ for sex, interventionalist versus noninterventionalist training, geographic location, and appointment level, respectively. There were differences in response rates among geographic regions, appointment level, and medical training. Junior and mid-career physicians were more likely to respond than senior physicians $(40.7 \%$ and $43 \%$ vs $25.5 \%$; $\mathrm{p}=0.004)$ and European participants were more likely to respond than participants from other geographic areas to the invitation $(67.9 \%$ vs $32.1 \%, p=0.001)$. Similarly, those with medical training in vascular stroke neurology and interventional neuroradiology had better response rates than their counterparts in interventional stroke neurology and diagnostic neuroradiology $(31.9 \%$ and $31.5 \%$ vs $19.3 \%$ and $13.4 \%$; $p=0.001$; table 2). There were no differences between early and late responders (online supplemental table 2).

\section{Physicians' judgments of acceptable uncertainty}

The distribution of respondent's judgements of the acceptable value for uncertainty is shown in figure 1 ; the median value was 3 out of 100 patients, with IQR $1-5$. The most chosen acceptable uncertainty was 5 out of 100 patients, with close to every third respondent (29.1\%) choosing this answer. A sensitivity analysis excluding respondents who defined values $>10$ as acceptable uncertainty $(n=6)$ did not change median or interquartile range (3, 1-5, respectively). When limiting the analysis to physicians with senior-level appointment $(n=126)$, acceptable uncertainty had the same values for median and interquartile range $(3,1-5$, respectively).

Distributions of acceptable uncertainty values by strata of sex, trial participation, training, and percentage of dedicated care time for patients with a stroke, are shown in the supplementary material (online supplemental figures 2-11). The median value of selected acceptable uncertainty values and IQR of all subgroups is displayed in online supplemental table 3. The most chosen answer was 5 out of 100 patients in all subgroups, except for respondents participating in IVT versus placebo trials (most chosen uncertainty: 1 out of 100 patients) and those with
Table 1 Respondents' baseline characteristics

\begin{tabular}{|c|c|}
\hline Characteristics & Overall \\
\hline $\mathrm{N}(\%)$ & $165(100)$ \\
\hline Male (\%) & $121(73.3)$ \\
\hline Training: interventionalist (\%) & $84(50.9)$ \\
\hline \multicolumn{2}{|l|}{ Geography (\%) } \\
\hline North America & $23(13.9)$ \\
\hline South America & $2(1.2)$ \\
\hline Europe & $112(67.9)$ \\
\hline Africa & $1(0.6)$ \\
\hline Middle East & $2(1.2)$ \\
\hline Asia & $10(6.1)$ \\
\hline Australia & $15(9.1)$ \\
\hline \multicolumn{2}{|l|}{ Appointment level (\%) } \\
\hline Junior & $11(6.7)$ \\
\hline Mid-career & $28(17.0)$ \\
\hline Senior & $126(76.4)$ \\
\hline \multicolumn{2}{|l|}{ Annual mechanical thrombectomy volume (\%) } \\
\hline$<100$ & $50(30.3)$ \\
\hline $100-200$ & $66(40.0)$ \\
\hline$>200-300$ & $29(17.6)$ \\
\hline$>300$ & $20(12.1)$ \\
\hline \multicolumn{2}{|l|}{ Dedicated stroke patient care time (\%) } \\
\hline$<10 \%$ & $10(6.1)$ \\
\hline $10-50 \%$ & $77(46.7)$ \\
\hline $51-99 \%$ & $63(38.2)$ \\
\hline $100 \%$ & $15(9.1)$ \\
\hline Acceptable uncertainty margin (\%), median (IQR)) & $3(1-5)$ \\
\hline \multicolumn{2}{|l|}{ Trial participation (\%) } \\
\hline IVT vs placebo: yes (\%) & $42(25.5)$ \\
\hline MT+IVT vs IVT: yes (\%) & $51(30.9)$ \\
\hline MT vs MT + IVT= :yes (\%) & $51(30.9)$ \\
\hline \multicolumn{2}{|l|}{ In patients undergoing MT do you stop IVT before full dose is administered? (\%) } \\
\hline $\begin{array}{l}\text { No, unless there are clear medical reasons (eg, angiographic signs } \\
\text { of bleeding) }\end{array}$ & $102(61.8)$ \\
\hline Yes, as a standard procedure at first device deployment & $16(9.7)$ \\
\hline Yes, as a standard procedure after successful reperfusion is reached & $7(4.2)$ \\
\hline $\begin{array}{l}\text { Yes, on an individual case basis after successful reperfusion is } \\
\text { reached }\end{array}$ & $31(18.8)$ \\
\hline Other & $9(5.5)$ \\
\hline
\end{tabular}

Data are displayed as $\mathrm{n}(\%)$, and median (IQR).

IVT, intravenous thrombolysis; MT, mechanical thrombectomy.

non-interventional training (most chosen uncertainty: 3 out of 100 patients).

On multivariable logistic regression median split analysis, interventional training was associated with higher values for acceptable uncertainty (aOR 2.20, 95\% CI 1.06 to 4.67), while senior appointment and annual number of mechanical thrombectomies in center $>200$ tended to be associated with lower uncertainty margins (aOR $0.27,95 \%$ CI 0.05 to 1.09 ; aOR 0.57 , $95 \%$ CI 0.25 to 1.25 , respectively), as presented in figure 2 . The directions of most associations were comparable when using first and fourth quartile splits, with participation in IVT versus placebo trials and participation in EVT versus IVT+EVT trials 


\begin{tabular}{|c|c|c|c|c|}
\hline Characteristics & Overall & Complete & $\begin{array}{l}\text { Incomplete and } \\
\text { non-responders }\end{array}$ & $P$ value \\
\hline N (\%) & 600 & 165 & 435 & \\
\hline Male (\%) & $445(74.2)$ & $121(73.3)$ & $324(74.5)$ & 0.855 \\
\hline Geography (\%) & & & & $<0.001$ \\
\hline North America & $132(22.0)$ & $23(13.9)$ & $109(25.1)$ & \\
\hline South America & $25(4.2)$ & $2(1.2)$ & $23(5.3)$ & \\
\hline Europe & $298(49.7)$ & 112 (67.9) & $186(42.8)$ & \\
\hline Africa & $17(2.8)$ & $1(0.6)$ & $16(3.7)$ & \\
\hline Middle East & $34(5.7)$ & $2(1.2)$ & $32(7.4)$ & \\
\hline Asia & $44(7.3)$ & $10(6.1)$ & $34(7.8)$ & \\
\hline Australia & $50(8.3)$ & $15(9.1)$ & $35(8.0)$ & \\
\hline $\begin{array}{l}\text { Appointment }(\%, \mathrm{n}=13 \\
\text { missing) }\end{array}$ & & & & 0.004 \\
\hline Junior & $27(4.6)$ & $11(6.7)$ & $16(3.8)$ & \\
\hline Mid-career & 65 (11.1) & $28(17.0)$ & $37(8.8)$ & \\
\hline Senior & $495(84.3)$ & $126(76.4)$ & $369(87.4)$ & \\
\hline Medical training (\%) & & & & 0.001 \\
\hline $\begin{array}{l}\text { Vascular stroke } \\
\text { neurologists }\end{array}$ & $272(45.3)$ & $87(52.7)$ & $185(42.5)$ & \\
\hline $\begin{array}{l}\text { Interventional stroke } \\
\text { neurologists }\end{array}$ & $31(5.2)$ & $6(3.6)$ & $25(5.7)$ & \\
\hline $\begin{array}{l}\text { Diagnostic } \\
\text { neuroradiologists }\end{array}$ & $119(19.8)$ & $16(9.7)$ & $103(23.7)$ & \\
\hline $\begin{array}{l}\text { Interventional } \\
\text { neuroradiologists }\end{array}$ & $178(29.7)$ & $56(33.9)$ & $122(28.0)$ & \\
\hline
\end{tabular}

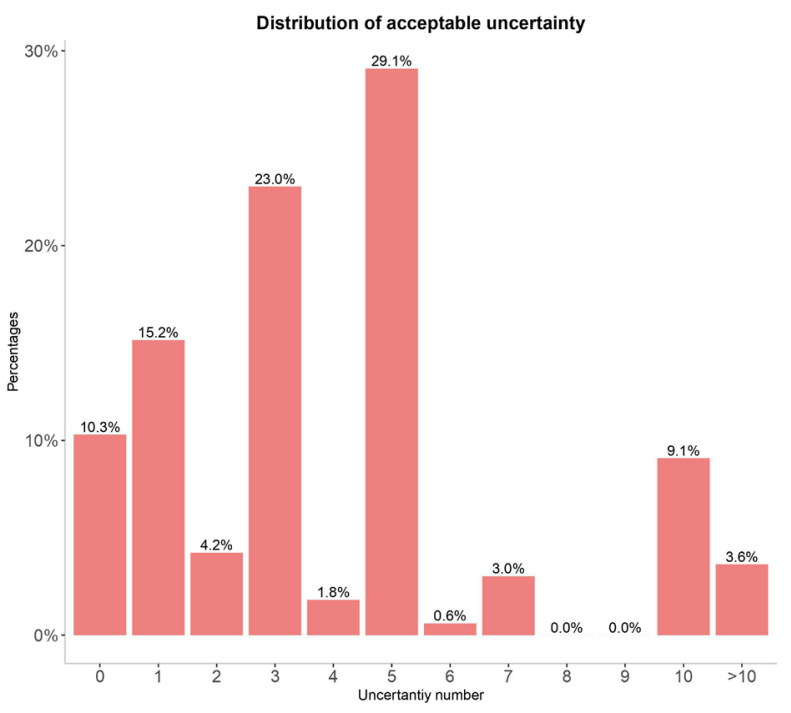

Figure 1 Distribution of respondents' acceptable uncertainty values. There was a right skewed distribution with the median response being 3 (IQR 1-5), indicating that most responders would skip the intravenous therapy and treat patients with endovascular intervention alone if effect point estimates were equal; either treatment could be a little better than the other; and, in the worst possible scenario, $3 \%$ of the patients receiving endovascular treatment alone would fail to regain functional independence due to skipping the intravenous therapy. Most respondents $(29.1 \%)$ selected $5 \%$ (mode) as acceptable uncertainty.

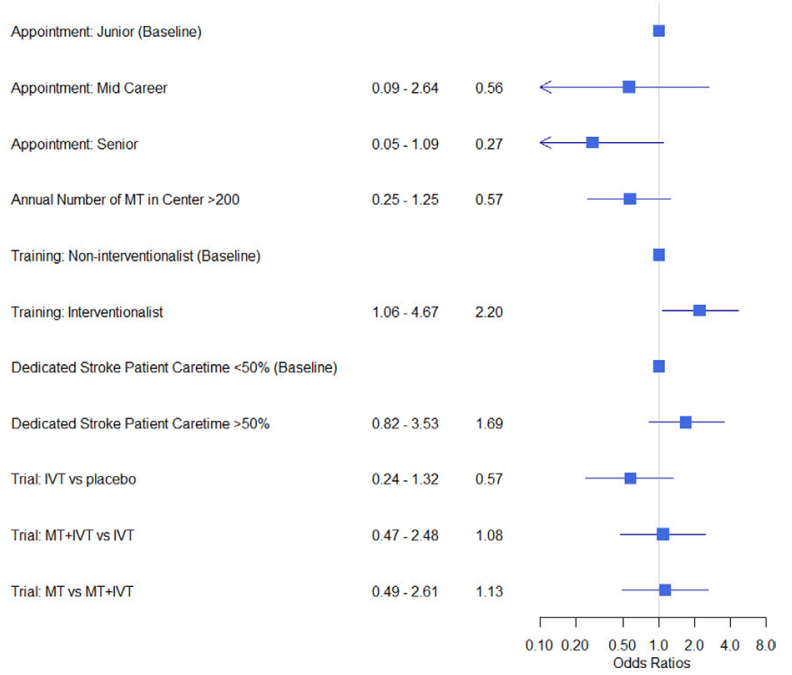

Figure 2 Forest plot showing effect of respondent characteristics on acceptable uncertainty values. Logistic regression analyses with dichotomized (median split) acceptable uncertainty margins defined as dependent functional outcome, were performed to evaluate associations between baseline characteristics and lower/higher chosen acceptable uncertainty margins. ORs $>1 /<1$ indicate association with higher/ lower acceptable uncertainty values, respectively. IVT, intravenous thrombolysis; MT, mechanical thrombectomy.

being associated with selecting lower acceptable uncertainty values on first and fourth quartile split analyses (online supplemental figure 5).

\section{DISCUSSION}

This survey found that physicians would generally feel comfortable skipping intravenous thrombolysis before mechanical thrombectomy in directly admitted patients at different degrees of certainty. For more than half of survey physicians, this certainty threshold was met if randomized trial data suggested that the number of patients achieving functional independence at 90 days was similar between both treatment groups, and one could be sufficiently sure that-in the worst case scenario-no more than 3/100 patients would not achieve functional independence at 90 days, owing to skipping intravenous thrombolysis. Moderate variance was noted among physicians as to what was considered an acceptable uncertainty, and there was weak evidence that uncertainty value selections differed with respect to training, case volume, and prior participation in specific clinical trials.

The findings of the current study are consonant with prior investigation. As expected, this study's value for acceptable uncertainty when assessing reasonable comparability (3\%) is modestly larger than the value for the MCID when assessing indistinguishability (1.3\%) found in a prior study using a superiority design scenario of a novel and safe neuroprotective agent. ${ }^{17}$ Although the sample size required to demonstrate noninferiority using a margin based on the MCID is unattainably large (18 205 patients per group for a study with 80\% power and $95 \%$ confidence), the sample size required to demonstrate non-inferiority based on a reasonable comparability margin is less extreme, though still sizeable (3419 patients per group).

The finding of a $3 \%$ reasonable comparability margin provides a useful comparison for non-inferiority margins employed in the six completed randomized trials of EVT alone versus 
IVT+EVT. ${ }^{9-15}$ Three of these trials used absolute risk difference margins $^{12} 1415$ and three relative risk difference margins. ${ }^{9-11} 13$ In the three trials using absolute risk difference, non-inferiority margins for functional independence at 3 months were defined as three to four times higher (ie, 10\% and 12\%) ${ }^{12} 1415$ than the median reasonable comparability margin found in this survey. The chosen absolute non-inferiority margin of $10 \%$ and $12 \%$ in those trials is equal to a preserved fraction of $60 \%$ and $51 \%$ of the endovascular treatment effect in the SWIFT PRIME trial, and 51\% and 41\% in the HERMES meta-analysis, respectively. ${ }^{1214151819}$ The other three trials, which used relative risk non-inferiority margins, were of generally similar magnitude to those used in the absolute risk margin trials. ${ }^{9-11} 13$ Importantly, each of the completed randomized controlled trials is thus not powered to fully assess reasonable comparability at a 3\% margin on their own. However, if individual trials or a meta-analytic aggregation of their findings, narrows the CI to below a $3 \%$ margin, then reasonable comparability can be demonstrated.

The current study employed survey question wording designed to elicit physician judgment of reasonable comparability (acceptable uncertainty) rather than indistinguishability (MCID) of treatments. In a prior survey assessing the MCID, the survey query was posed in a superiority frame, asking physicians to choose the smallest difference in outcomes that would lead them to use a simply delivered drug therapy in clinical practice. ${ }^{17}$ In addition, the query asked physicians to consider treatment effects within a denominator of 1000 patients in order to allow greater resolution in specifying small values. In contrast, in the current study, the survey query was posed in a non-inferiority frame, asking what uncertainty level physicians would judge sufficient to consider a less complicated rather than more complicated treatment strategy (ie, skipping IVT before EVT). With this approach, physicians indicated the maximally acceptable potential risk that patients may have a poorer outcome due to their treatment decision, although the framework of the survey implies that their decision may also be equal to, or more beneficial than, the reference treatment. In addition, the query asked physicians to consider treatment effects within a denominator of 100 and specifically permitted ' 0 ' as a possible answer. This allows for the option that physicians will only skip IVT if there is virtually no uncertainty around the interchangeability of treatments, which would mean that the lower bound of the $95 \%$ confidence would just cross or not cross $0 \%$, formally representing superiority of the EVT alone approach.

In the present study, physicians trained in non-interventional specialties selected more stringent acceptable uncertainty values than did physician interventionalists. This difference may in part reflect each group's familiarity with, and allegiance toward, the treatment modality they themselves deliver: IVT therapy for non-interventionalists and EVT for interventionalists. Moreover, it may reflect the thought process of interventionalists administering dual or single antiplatelets during the intervention in cases which require cervical stenting. In the setting of heightened risk of hemorrhage, they may tend to accept a higher level of uncertainty. Also, interventionalist physicians may generally be characterologically more accepting of risk than non-interventionalists. ${ }^{20}$

\section{Limitations}

This study has some limitations. First, selection of participants was based on publicly available institutional website information, publications, and participation in trials, rather than a random sample of all stroke physicians. The response rate was moderate, and senior physicians and European physicians were over-represented among respondents compared with their numbers in actual practice. Second, as with all surveys, this survey was subject to anchoring, centrality, and framing bias. Figural examples of response options were included to maximize understanding, and examples can exert anchoring bias, but the lack of response peaks at example values suggests this did not occur. Third, use of a reference group size of 100 might have modestly increased risk taking by contextual group size differences, as it has been shown that risk-seeking behavior is generally greater if group sizes are presented in small numbers in experimental lifedeath decision problems. ${ }^{21} 22$ Fourth, we were unable to assess the actual knowledge of, and familiarity with, non-inferiority trials of the survey participants, which might have influenced their decision and comprehension of the survey question. Last, there are several key points considered in a non-inferiority trial, including disease prevalence, practicality, and cost-effectiveness, which were not fully incorporated into the given scenario. We intentionally kept the factor of cost out of the equation, since economic considerations are probably affected by location and time. As such, it could introduce unnecessary heterogeneity, limit generalizability of the study results, and might have confounded clinical judgment. ${ }^{23}$

\section{CONCLUSION}

This study found that international expert stroke noninterventional and interventional physicians would consider direct EVT reasonably comparable to IVT+EVT and be willing to treat patients admitted to comprehensive stroke center with direct EVT at different certainty thresholds. Most physicians would treat with direct EVT if the following criteria were met: randomized trials showed the same point estimates for functional independence outcome, and the confidence intervals excluded the possibility that no more than $3 / 100$ patients would not regain functional independence due to skipping the drug. Non-interventional physicians were modestly more conservative than interventional physicians in the degree of uncertainty they would find acceptable. This value for acceptable uncertainty based on survey data from a predominantly European group of international stroke experts may be useful for selecting the noninferiority margin when meta-analysis is undertaken pooling data from all completed trials.

\section{Author affiliations}

${ }^{1}$ Department for Diagnostic and Interventional Neuroradiology, Inselspital University Hospital Bern, Bern, Switzerland

${ }^{2}$ Department of Neurology, Inselspital University Hospital Bern, Bern, Switzerland

${ }^{3}$ Department of Radiology and Nuclear Medicine, Amsterdam University Medical Centres, Amsterdam, Noord-Holland, The Netherlands

${ }^{4}$ Neurovascular Center, Naval Medical University, Shanghai, China

${ }^{5}$ Department of Neurology, Army Medical University Xinqiao Hospital, Chongqing, Sichuan, China

${ }^{6}$ Department of Neurology, Grady Memorial Hospital, Chickasha, Oklahoma, USA ${ }^{7}$ Department of Neurology, Nippon Medical School, Bunkyo-ku, Tokyo, Japan

${ }^{8}$ Division of Stroke Prevention and Treatment, Faculty of Medicine, University of Tsukuba, Tsukuba, Ibaraki, Japan

${ }^{9}$ Melbourne Brain Centre, The Royal Melbourne Hospital, Parkville, Victoria, Australia

${ }^{10}$ Department of Radiology, The Royal Melbourne Hospital, Parkville, Victoria,

Australia

${ }^{11}$ Department of Interventional Neuroradiology, Beijing Tiantan Hospital, Beijing, China

${ }^{12}$ Department of Neurology, Amsterdam University Medical Centres, Amsterdam, The Netherlands

${ }^{13}$ Department of Neurology, UCLA, Los Angeles, California, USA

${ }^{14}$ Comprehensive Stroke Center and Neurology, David Geffen School of Medicine, Los Angeles, California, USA

Twitter Adnan Mujanovic @adnan_mujanovic and Urs Fischer @FishingNeurons Contributors JK and AM contributed to conception, design, data acquisition, 
analysis, interpretation, and writing of the publication. JK is the guarantor of the study. KT and MK contributed to data interpretation and critical revision of the manuscript. TRM contributed to data acquisition and critical revision of the manuscript. YBWEMR contributed data analysis and interpretation, and writing of the publication. CBLMM contributed towards design and writing of the first draft. JG contributed towards data collection, data interpretation, and critical manuscript revision. JLS and UF contributed to conception and design, critical revision of the publication for important intellectual content, and supervision. All other authors contributed to interpretation of the data, and critical revision of the manuscript for important intellectual content.

Funding The authors have not declared a specific grant for this research from any funding agency in the public, commercial or not-for-profit sectors.

Competing interests CBLMM reports grants from CVON/Dutch Heart Foundation, TWIN Foundation, European Commission, Health Evaluation Netherlands, and Stryker outside the submitted work (all paid to institution); and is shareholder of Nico-lab. YBWEMR is a shareholder of Nico-lab. UF reports grants during the conduct of the study from Medtronic, Stryker, and CSL Behring, unrelated to the submitted work, and his board membership at the Journal of Neurolnterventional Surgery. JG is a global principal investigator of STAR (Solitaire FR Thrombectomy for Acute Revascularisation), Clinical Event Committee member of the PROMISE study (Prospective, Multicenter, Observational, Single-Arm European Registry on the ACE Reperfusion Catheters and the Penumbra System in the Treatment of Acute Ischemic Stroke; Penumbra), and a principal investigator and consultant for the SWIFT DIRECT study (Solitaire With the Intention for Thrombectomy Plus Intravenous tPA Versus DIRECT Solitaire Stent-Retriever Thrombectomy in Acute Anterior Circulation Stroke; Medtronic) and receives Swiss National Science Foundation grants for magnetic resonance imaging in stroke. JK reports grants from the Swiss Academy of Medical Sciences/Bangerter Foundation, Swiss Stroke Society, Swiss National Science Foundation, and the Clinical Trials Unit Bern during the conduct of this study.

Patient consent for publication Not applicable.

Ethics approval This study does not involve human participants.

Provenance and peer review Not commissioned; externally peer reviewed.

Data availability statement Data are available upon reasonable request. Data are available from the corresponding author upon a reasonable request, together with a research plan proposal.

Supplemental material This content has been supplied by the author(s). It has not been vetted by BMJ Publishing Group Limited (BMJ) and may not have been peer-reviewed. Any opinions or recommendations discussed are solely those of the author(s) and are not endorsed by BMJ. BMJ disclaims all liability and responsibility arising from any reliance placed on the content. Where the content includes any translated material, BMJ does not warrant the accuracy and reliability of the translations (including but not limited to local regulations, clinical guidelines, terminology, drug names and drug dosages), and is not responsible for any error and/or omissions arising from translation and adaptation or otherwise.

Open access This is an open access article distributed in accordance with the Creative Commons Attribution Non Commercial (CC BY-NC 4.0) license, which permits others to distribute, remix, adapt, build upon this work non-commercially, and license their derivative works on different terms, provided the original work is properly cited, appropriate credit is given, any changes made indicated, and the use is non-commercial. See: http://creativecommons.org/licenses/by-nc/4.0/.

ORCID iDs

Johannes Kaesmacher http://orcid.org/0000-0002-9177-2289

Adnan Mujanovic http://orcid.org/0000-0002-6839-7134

Thomas R Meinel http://orcid.org/0000-0002-0647-9273

\section{REFERENCES}

1 Kaul S, Diamond GA. Good enough: a primer on the analysis and interpretation of noninferiority trials. Ann Intern Med 2006;145:62.

2 Rehal S, Morris TP, Fielding K, et al. Non-Inferiority trials: are they inferior? A systematic review of reporting in major medical journals. BMJ Open 2016;6:e012594.

3 Althunian TA, de Boer A, Groenwold RHH, et al. Defining the noninferiority margin and analysing noninferiority: an overview. Br I Clin Pharmacol 2017;83:1636-42.

4 Althunian TA, de Boer A, Klungel $\mathrm{OH}$, et al. Methods of defining the non-inferiority margin in randomized, double-blind controlled trials: a systematic review. Trials 2017;18:107.

5 Lange S, Freitag G. Special invited papers section: therapeutic equivalence - clinical issues and statistical methodology in noninferiority trials. Biom. J. 2005;47:12-27.

6 Tsui M, Rehal S, Jairath V, et al. Most noninferiority trials were not designed to preserve active comparator treatment effects. I Clin Epidemio/ 2019;110:82-9.

7 Lin C-J, Saver JL. Noninferiority margins in trials of thrombectomy devices for acute ischemic stroke: is the bar being set too low? Stroke 2019;50:3519-26.

8 McGlothlin AE, Lewis RJ. Minimal Clinically Important Difference: Defining What Really Matters to Patients. In: Livingston EH, Lewis RJ, eds. JAMA guide to statistics and methods. New York, NY: McGraw-Hill Education, 2019.

9 LeCouffe NE, Kappelhof M, Treurniet KM, et al. A randomized trial of intravenous alteplase before endovascular treatment for stroke. N Engl J Med 2021;385:1833-44.

10 Yang $P$, Zhang $Y$, Zhang L, et al. Endovascular thrombectomy with or without intravenous alteplase in acute stroke. N Engl J Med 2020;382:1981-93.

11 Suzuki K, Matsumaru Y, Takeuchi M, et al. Effect of mechanical thrombectomy without vs with intravenous thrombolysis on functional outcome among patients with acute ischemic stroke: the SKIP randomized clinical trial. JAMA 2021;325:244-53.

12 Zi W, Qiu Z, Li F, et al. Effect of endovascular treatment alone vs intravenous alteplase plus endovascular treatment on functional independence in patients with acute ischemic stroke: the DEVT randomized clinical trial. JAMA 2021;325:234-43.

13 Treurniet KM, LeCouffe NE, Kappelhof M, et al. Mr CLEAN-NO IV: intravenous treatment followed by endovascular treatment versus direct endovascular treatment for acute ischemic stroke caused by a proximal intracranial occlusion-study protocol for a randomized clinical trial. Trials 2021;22:141.

14 Fischer U, Kaesmacher J, S Plattner P, et al. Swift direct: Solitaire ${ }^{T M}$ with the intention for thrombectomy plus intravenous t-PA versus direct Solitaire ${ }^{T M}$ Stent-retriever thrombectomy in acute anterior circulation stroke: methodology of a randomized, controlled, multicentre study. Int I Stroke 2021:174749302110487.

15 Mitchell PJ, Yan B, Churilov L, et al. DIRECT-SAFE: a randomized controlled trial of direct endovascular clot retrieval versus standard bridging therapy. J Stroke 2022;24:57-64.

16 Wangge $\mathrm{G}$, Klungel $\mathrm{OH}$, Roes $\mathrm{KCB}$, et al. Room for improvement in conducting and reporting non-inferiority randomized controlled trials on drugs: a systematic review. PLoS One 2010;5:e13550.

17 Cranston JS, Kaplan BD, Saver JL. Minimal clinically important difference for safe and simple novel acute ischemic stroke therapies. Stroke 2017;48:2946-51.

18 Saver JL, Goyal M, Bonafe A, et al. Stent-retriever thrombectomy after intravenous t-PA vs. t-PA alone in stroke. N Eng/ J Med 2015;372:2285-95.

19 Goyal M, Menon BK, van Zwam WH, et al. Endovascular thrombectomy after large-vessel ischaemic stroke: a meta-analysis of individual patient data from five randomised trials. Lancet 2016;387:1723-31.

20 Surbeck W, Samuel R, Spieler D, et al. Neurologists, neurosurgeons, and psychiatrists' personality traits: a comparison. Acta Neurochir 2020;162:461-8.

21 Wang XT. Domain-specific rationality in human choices: violations of utility axioms and social contexts. Cognition 1996;60:31-63.

22 Wang XT. Framing effects: dynamics and task domains. Organ Behav Hum Decis Process 1996;68:145-57.

23 Ospel JM, McDonough R, Kunz WG. Is concurrent intravenous alteplase in patients undergoing endovascular treatment for large vessel occlusion stroke cost-effective even if the cost of alteplase is only US\$1? J Neurointerv Surg 2021:1-5. 